\title{
Transparenz für Patienten: Qualitätsergebnisse im AOK-Krankenhausnavigator
}

\section{Einleitung \\ $\nabla$}

Informationen zur Qualität stationärer Leistungen sind für Patienten und Krankenhaus-Einweiser von besonderem Interesse, wenn vor einer Behandlung eine Klinik mit hoher Qualität ausgewählt werden soll. Patienten äußern einen großen Informationsbedarf und die Informationsangebote haben sich in den letzten Jahren in Deutschland grundsätzlich verbessert - wozu die verpflichtende Veröffentlichung der strukturierten Qualitätsberichte nach §137 SGB V durch die Kliniken und die Klinikrechercheportale im Internet beigetragen haben -, dennoch wird bei der Kliniksuche zumeist auf Empfehlungen von Freunden und Bekannten oder Ärzten zurückgegriffen [1]. Eine Ursache dafür ist, dass Daten zur Ergebnisqualität von Klinikbehandlungen in Deutschland kaum öffentlich verfügbar sind, obwohl Behandlungserfolg und Komplikationshäufigkeit für Patienten zu den wichtigsten Entscheidungskriterien gehören. Dabei ist eine öffentliche Berichterstattung über Behandlungsergebnisse (z.B. in Form von „report cards“) international üblich $[2,3]$.

\section{Langzeitindikatoren im Verfahren „Qualitätssicherung mit Routinedaten“" (QSR) \\ $\checkmark$}

Die Qualitätsmessung durch das QSR-Verfahren legt nun gerade den Fokus auf Ergebnisqualität. Dafür werden Ereignisse während und nach dem aktuellen Krankenhausaufenthalt ausgewertet und in der Bewertung berücksichtigt. Auf der Grundlage von Routinedaten aus Krankenhausabrechnung und Krankenkassendaten ist eine Langzeitbeobachtung möglich, wobei gleichzeitig zusätzliche Dokumentationsaufwände vermieden werden.

QSR zeigt auf, dass zwischen den Kliniken relevante Qualitätsunterschiede bestehen. Beispielsweise kommt es beim Einsatz eines künstlichen Hüftgelenks bei AOK-Patienten in den Jahren 2006 bis 2008 innerhalb von 12 Monaten nach Operation durchschnittlich bei $4 \%$ der Patienten zu einer Revisionsoperation. Bei einem Viertel der Kliniken ist die Revisionsrate um mindestens $50 \%$ erhöht (5,9\% oder höher).

\author{
QSR für Patienten im \\ AOK-Krankenhausnavigator \\ $\nabla$
}

Seit April 2010 werden Daten zur Behandlungsqualität von Kliniken mittels QSR-Verfahren im Klinik-Suchportal der AOK veröffentlicht. Der AOK-Krankenhausnavigator auf Basis der Weißen Liste stellt die Informationen aus den gesetzlichen Qualitätsberichten zusammen und ergänzt für endoprothetische Leistungen (Hüftund Kniegelenk-Ersatz sowie Oberschenkelbrüche) die wichtigsten QSR-Indikatoren. Die risikoadjustierten Kennzahlen aus dem QSR-Verfahren werden als Werte der standardisierten Mortalitätsrate (SMR) angegeben. Die Klinikergebnisse sind in drei Kategorien eingeteilt, je nachdem ob das Konfidenzintervall (KI) der Komplikationsrate über, unter oder im Durchschnitt aller deutschen Kliniken liegt: Krankenhäuser im untersten Quintil der KI-Obergrenzen der SMR weisen mit großer Sicherheit vergleichsweise geringe Komplikationsraten auf, Krankenhäuser im obersten Quintil der KI-Untergrenzen hingegen vergleichsweise hohe Raten. Eine Abwertung unterbleibt bei weniger als 5 Komplikationen. Alle übrigen Krankenhäuser werden dem Bereich durchschnittlicher Qualität zugerechnet. Die Berichterstattung erfolgt auf der Grundlage von Krankenhausbehandlungen von AOK-Patienten aus 3 Jahren für Kliniken mit mehr als $30 \mathrm{Be}-$ handlungsfällen.

Die Veröffentlichung hat zu einem breiten Medienecho geführt. Nachdrucke von QSR-Kliniklisten in den Medien und hohe Zugriffszahlen im AOK-Krankenhausnavigator belegen das Interesse der Öffentlichkeit an Qualitätsinformationen über Krankenhausbehandlungen. Das Angebot soll auf weitere Indikationen erweitert werden.

Autorenerklärung: Der Autor ist im Wissenschaftlichen Institut der AOK (WIdO) beschäftigt.

\section{Literatur}

1 Geraedts $M$, de Cruppé $W$. Wahrnehmung und Nutzung von Qualitätsinformationen durch Patienten. In: Klauber J, Geraedts M, Friedrich J, Wasem J (Hrsg). Krankenhaus-Report 2011. Stuttgart: 2011

2 Matthes N, Wiest A. Veröffentlichung von Qualitätsdaten für Krankenhäuser in den USA, In: Klauber J, Robra BP, Schellschmidt H (Hrsg). Krankenhaus-Report 2004. Stuttgart: 2005

3 Ferris TG, Torchiana DF. Public release of clinical outcomes data-online CABG report cards. N Engl J Med 2010; 363: 1593-1595
C. Günster

Qualitätsmanagement

Schlüsselwörter Öffentliche

Berichterstattung

hospital report cards

Ergebnisqualität

Keywords

public reporting

hospital report cards

outcome assessment

Institut

Wissenschaftliches Institut der AOK (WIdO), Berlin

Bibliografie

DOI 10.1055/s-0031-1286080

Dtsch Med Wochenschr 2011 ;

136: S51 - (c) Georg Thieme

Verlag KG Stuttgart · New York . ISSN 0012-0472

Korrespondenz Christian Günster

Wissenschaftliches Institut der AOK (WIdO)

Rosenthaler Str. 31

10178 Berlin

eMail christian.guenster@

wido.bv.aok.de 